\title{
Upgrade of GLE database: assessment of effective dose rate at flight altitude
}

\author{
S. Tuohino ${ }^{\text {a }}$ A. Ibragimov ${ }^{b}$ I. Usoskin ${ }^{\mathrm{c}, \mathrm{d}}$ A. Mishev ${ }^{\mathrm{c}, \mathrm{d}, *}$

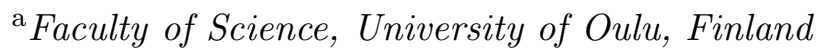 \\ ${ }^{\mathrm{b}}$ Independent researcher, Helsinki, Finland \\ ${ }^{\mathrm{c}}$ Space Climate Research Unit, University of Oulu, Finland \\ d Sodankylä Geophysical Observatory (Oulu unit), University of Oulu, Finland
}

\begin{abstract}
A new database for assessment of radiation doses in the Earth atmosphere, related to GLE events is created under VarSiTi/SCOSTEP support and incorporated to the International ground level enhancement (GLE) database (gle.oulu.fi). The upgraded database provides, for each GLE event, where possible, information on the estimated energy/rigidity spectra of solar energetic particles and the corresponding computed effective doses at cruise flight altitude of $35 \mathrm{kft}$ (10668 m above sea level). The computations are performed for various reconstructions of solar energetic particles spectra, available in literature, thus for some events there are several results. Computations were performed using a recent model for assessment of effective dose due to cosmic ray particles, applied specifically in the polar region, where the exposure is maximal. This upgrade allows one to estimate the radiation effects at cruise flight altitude caused by major GLE events over several decades.
\end{abstract}

Key words: Solar energetic particles, GLE events, GLE database, radiation environment

\section{Introduction}

The assessment of aircrew exposure to radiation due to cosmic rays (CRs), specifically during ground level enhancement (GLE) events is an important

\footnotetext{
* Corresponding author:A.Mishev, Space Climate Research Unit, University of Oulu, Finland; Tel:(+358)44 9160753

Email address: alexander.mishev@oulu.fi, alex_mishev@yahoo.com (A. Mishev).
} 
topic in the field of space weather (e.g. Baker, 1998; Lilensten and Bornarel, 2009; Vainio et al., 2009, and references therein). Thus, several recommendations and regulations appeared, where the exposure of flying personnel to the cosmic radiation is recommended to be regarded as an occupational (ICRP, 1991, 2007). Accordingly in EU, it was suggested to assess the individual accumulated doses of cockpit and cabin crew (EURATOM, 1996, 2013).

The assessment of the radiation exposure at typical flight altitudes due to $\mathrm{CR}$ of galactic and/or solar origin is not a simple task, because it depends on a geographic position and altitude (the intensity and energy distribution of the secondary CR particles), solar activity, geomagnetic conditions as well as on the eventual occurrence of solar energetic particle (SEP) events (Spurny et al., 1996, 2002; Shea and Smart, 2000). Moreover, the latter posses random occurrence and large variability. It is known that the main source of particles determining the radiation field at flight altitudes are CRs, specifically those originating from the Galaxy (e.g. O'Brien, 1970). According to the current knowledge, the majority of CRs originate from the Galaxy, the bulk are protons and $\alpha$ - particles with small abundance of heavier nuclei (e.g. Gaisser and Stanev, 2010, and references therein). Primary energetic CR particles enter in the atmosphere, collide with nuclei of the ambient air and produce certain number of secondary particles, which are eventually stopped and/or also collide, thus leading to the development of an extensive air shower. Occasionally, the Sun emits high energy particles (e.g. Reames, 2013; Klein and Dalla, 2017, and references therein). In some cases the energy of SEPs is GeV/nucleon, which is enough to produce an atmospheric shower registered by ground based detectors, specifically neutron monitors (NMs), an event known as GLE (e.g. Shea and Smart, 1982; Desai and Giacalone, 2016). A developed over the years database provides information of NM count rates around the globe during GLEs. It was developed by the research community e.g. L. Gentile, M. Shea and D. Smart, M. Duldig, presently hosted by the University of Oulu.

Both, GCR and SEPs, producing large diversity of secondary particles, determine the complex radiation field at flight altitudes. It is obvious that GLE events could enhance considerably the radiation exposure at flight altitudes (e.g. Spurny et al., 2002). Besides, GLE events differ from each other in spectra, angular distribution and duration as well as dynamics of characteristics governing the radiation exposure (Gopalswamy et al., 2012; Moraal and McCracken, 2012). Therefore, the assessment of the radiation exposure of aircrew due to SEP event is normally performed retrospectively on the basis of retrieved information form ground based detectors, e.g. NMs or rarely from in-situ measurements (e.g. Spurny and Dachev, 2001; Getley, 2004). 
In order to assess the radiation exposure hazard due to CRs, specifically during GLE events, it is necessary to possess precise and correct information about their energy and angular distribution. It was recently shown that the radiation exposure at aviation altitudes can be estimated using Monte Carlo simulations and/or transport models. As a result several models appeared (e.g. Ferrari et al., 2001; Getley et al., 2005a; Sato et al., 2008; Matthiä et al., 2008; Latocha et al., 2009; Mertens et al., 2013; Al Anid et al., 2014; Kataoka et al., 2014; Mishev et al., 2014a; Copeland, 2017; Hands et al., 2017) and good agreement between several models is observed (Bottollier-Depois et al., 2009). Here we compute the effective dose rate during several GLE events, where the information is available, at cruise flight altitude of $35 \mathrm{kft}$ $(10668 \mathrm{~m})$ in a region with low cut-off rigidity, namely with $R_{c}<1$ GV. For the computations we employed a recently proposed model for computation of effective dose (Mishev and Usoskin, 2015).

2 Model for computation of effective dose rate at aviation altitudes

Herein, we employed numerical model for computation of effective dose at aviation altitudes. The full description of the model with the corresponding look-up tables and comparison with reference data is given elsewhere (Mishev and Usoskin, 2015).

The model is based on pre-computed effective dose yield functions, the latter is the response in the sense of effective dose, to the monoenergetic unit flux of primary particle entering the Earth's atmosphere, derived with high statistics Monte Carlo simulations. The effective dose rate at a given atmospheric depth $h$ induced by primary CR particles is computed using the expression:

$$
E\left(h, R_{c}, \theta, \varphi\right)=\sum_{i} \int_{E_{c u t}, i}^{\infty} \int_{\Omega} J_{c}\left(T^{\prime}\right) Y_{i}\left(T^{\prime}, h\right) d \Omega d T^{\prime}
$$

where $h$ is the altitude above sea level, $R_{c}$ is the local cut-off rigidity, $\theta$ and $\varphi$ are the angles of incidence of the arriving particle, $J_{i}\left(T^{\prime}\right)$ is the differential energy spectrum of the primary $\mathrm{CR}$ arriving at the top of the atmosphere for $i$ component (proton and/or $\alpha$-particle) and $Y_{i}$ is the effective dose yield function for the corresponding primary CR component. In Eq. (1) the integration is over the kinetic energy above $E_{\text {cut }}\left(R_{c}\right)$, which is defined by the local cut-off rigidity $R_{c}$ for a nuclei of type $i$ at a given geographic location by the 
expression:

$$
E_{c u t, i}=\sqrt{\left(\frac{Z_{i}}{A_{i}}\right)^{2} R_{c}^{2}+E_{0}^{2}}-E_{0}
$$

where $E_{0}=0.938 \mathrm{GeV} / \mathrm{c}^{2}$ is the proton's rest mass.

Accordingly, the yield function $Y_{i}$ is defined as

$$
Y_{i}\left(T^{\prime}, h\right)=\sum_{j} \int_{T^{*}} F_{i, j}\left(h, T^{\prime}, T^{*}, \theta, \varphi\right) C_{j}\left(T^{*}\right) d T^{*}
$$

where $C_{j}\left(T^{*}\right)$ is the fluence to effective dose conversion coefficient for a secondary particle of type $j$ (neutron, proton, $\gamma, e^{-}, e^{+}, \mu^{-}, \mu^{+}$, $\left.\pi^{-}, \pi^{+}\right)$with energy $T^{*}, F_{i, j}\left(h, T^{\prime}, T^{*}, \theta, \varphi\right)$ is the secondary particle fluence of type $j$, produced by a primary particle of type $i$ (proton and/or $\alpha$-particle) with a given primary energy $T^{\prime}$. The conversion coefficients $C_{j}\left(T^{*}\right)$ are considered according to Petoussi-Henss et al. (2010).

For GCR Eq.1-3 lead to

$$
E=4 \pi^{2}\left[\int_{E_{c u t}}^{\infty} J_{p}\left(T^{\prime}\right) Y_{p}\left(T^{\prime}\right) d T^{\prime}+\int_{E_{c u t}}^{\infty} J_{\alpha}\left(T^{\prime}\right) Y_{\alpha}\left(T^{\prime}\right) d T^{\prime}\right]
$$

The latter equation possess two integral terms, first describing the contribution of $\mathrm{CR}$ protons, while the second accounts $\alpha$-particles and includes also heavier nuclei similarly to Usoskin and Kovaltsov (2006); Mishev and Velinov (2011). In case of GLE events, the effective dose is a superposition of GCR contribution (both integrands of Eq.4) and SEPs, the latter is computed using only the first term in Eq.4, assuming the corresponding SEP spectra. Note, that here we consider GLE spectra derived mostly on the basis of NM records (Table 1), the latter being an integral detector and not providing information about the mass composition. For GCRs we employ the force field model (Gleeson and Axford, 1968; Burger et al., 2000; Usoskin et al. , 2005) with the corresponding parametrization of local interstellar spectrum according to Usoskin and Kovaltsov (2006).

The differential intensity $J_{i}\left(T^{\prime}\right)$ of cosmic ray nuclei of type $i$ at 1 $\mathrm{AU}$ is given as

$$
J_{i}\left(T^{\prime}, \phi\right)=J_{L I S, j}\left(T^{\prime}+\Phi_{j}\right) \frac{\left(T^{\prime}\right)\left(T^{\prime}+2 T_{r}\right)}{\left(T^{\prime}+\Phi_{j}\right)\left(T^{\prime}+\Phi_{j}+2 T_{r}\right)}
$$


where $T^{\prime}$ is the kinetic energy per nucleon of primary CR with charge $\mathbf{Z}$ and atomic mass $\mathbf{A}$ and $\Phi_{i}=\left(Z_{i} e / A_{i}\right) \phi$. The only parameter of this model is the modulation potential $\phi$ given in units of MV, which explicitly accounts the solar activity. Herein, the modulation potential is considered according to Usoskin et al. (2011). The flux of incoming particles is assumed to be isotropic, which is conservative approach for GLE particles in sense of radiation exposure.

3 Assessment of effective dose rate at aviation altitude during GLEs

There are 72 GLE events so far, the last recorded by the global NM network on 10 September 2017. Their occurrence rate is roughly one per year (Shea and Smart, 1990; Stoker, 1995; Gopalswamy et al., 2012). Here we compute the effective dose rate for each GLE event, where possible, using various sets of derived energy/rigidity spectra and employing the model described in Section 2. The computations are performed for illustration, at typical cruise flight altitude of $35 \mathrm{kft}$ $(10668 \mathrm{~m})$, which can be used as a reference, and in a region with low cut-off rigidity $R_{c}<1 \mathrm{GV}$, where the radiation exposure is maximal. The results are shown for various reconstructions of SEPs spectra, available in literature, thus for some events there are several results. The full list of the events considered in this study with the corresponding bibliography is given in Table 1.

The assessed effective dose rate varies from several $\mu \mathrm{Sv} . \mathrm{h}^{-1}$, with small contribution of SEPs to tens and/or hundreds $\mu \mathrm{Sv} \cdot \mathrm{h}^{-1}$. During the strongest recorded events (e.g. GLE 5 and GLE 69) the estimated dose rate is about a $1-3.5 \mathrm{mSv} \cdot \mathrm{h}^{-1}$ (Table 2). Therefore, during the strongest events it would be possible to receive over one hour flight in the polar region, an exposure comparable to the annual recommended for the population (EURATOM, 2013). The described computations are released as a new database for assessment of radiation exposure in the Earth atmosphere, related to GLE events, which is supplementary and now linked to the International GLE database gle.oulu.fi (Usoskin et al., 2015). Hence, the new database provides, for each GLE event, where possible, an illustrative information for the energy/rigidity spectra of solar energetic particles and the corresponding computed effective doses for several periods of the event. Examples are shown for GLE 59 (Fig.1), GLE 60 (Fig.2) and GLE 70 (Fig.3). One can see that for GLE 59 are three different sets of derived spectra (the references given at the bottom), resulting on three different sets of computed effective dose. 
An example of the used SEP spectra is given in Fig.4a, which corresponds to set 3 (Mishev and Usoskin, 2016), accordingly Fig.4b, which corresponds to set 1 (Bombardieri et al., 2006). For GLE 60, there is only one set of SEP spectra (Fig. 5) (Bombardieri et al., 2007), while for GLE 70 (Fig.3) there are five different sets. An example for the used SEP spectra in the latter case is given in Fig.6a (set 4) and Fig.6b (set 3). The hardest SEP spectra resulting on a maximum peak exposure during GLE 69 are shown in Fig. 7 (Bombardieri et al., 2008). For several events is given the time interval over the spectra are integrated, e.g. set 3 (Fig.4a) for GLE 59 (Fig. 1). When the time interval is uncertain or not available, the interval end is not given. For all events is also computed the contribution of GCRs to the effective dose using the corresponding modulation potential, without considering possible Forbush decrease, which can be neglected during GLEs as a conservative approach. Details of the peak effective dose rates, contribution of GCRs to the exposure and the corresponding modulation potential are given in Table 2. Note, that the computed effective doses differ from each other for the same event assuming different derived spectra as reported in Bütikofer and Flückiger $(2013,2015)$. This is due to differences in GLE reconstructions, specifically the particle intensity as well as rigidity and spectral shapes. A detailed study of the impact of various sets of SEP spectra within one event on radiation exposure at flight altitude is beyond the scope of this work. The full list of computations is available in http://gle.oulu.fi/\#/dose.

\section{Discussion and Summary}

During strong GLE events, passengers and aircrew may receive radiation exposure doses above the background level due to GCR. Therefore, since the exposure of flying personnel to cosmic radiation is regarded as occupational, it should be assessed and monitored. While the background radiation at aviation altitudes could be routinely monitored nowadays and/or easy to assess by computations and/or using data sets with corresponding measurements, the estimation of radiation doses due to SEPs poses a significant challenge because of their random occurrence and large variability. Normally it is assessed retrospectively, when the necessary GLE particles characteristics are available. Obviously a systemic study of the radiation exposure during GLE events is necessary, specifically at polar region where it would be maximal.

Herein, we performed computations using a recent model for assessment of effective dose due to CRs at aviation altitude, we esti- 
mated the radiation exposure during GLE events and created the corresponding database. During the computations a conservative approach of SEPs angular distribution is assumed, namely an isotropic. The mass composition of SEPs is assumed to be protons. The contribution of heavier nuclei can be assessed, when the appropriate information about SEP mass composition is available, similarly to Xapsos et al. (2007). However, taking into account the typical mass composition of SEPs and their energy range, the contribution of heavier than proton particles to the radiation exposure at flight altitudes can be quantitatively considered as marginal.

The new database is now linked to the existing GLE database http://gle. oulu.fi/\#/dose.The database will be kept updated when new events occur and/or new information for historical events is retrieved as well as improvement of the model computations. Hence, the new database provides, for each observed GLE event where data are available, information on the spectral properties of SEPs and the corresponding effective doses at typical cruise altitude of $35 \mathrm{kft}$. This allows one to estimate the radiation effects during GLE events over several solar cycles and give good basis for subsequent comparison between models and measurements (Getley et al., 2005b; Beck et al., 2006; Ploc et al., 2013). A more detailed computation, namely at several flight altitudes, time intervals, considering anisotropy affects is planified as forthcoming work.

\section{Acknowledgements}

This work was supported by VarSITI Program of ICSU Scientific Committee on Solar-Terrestrial Physics (SCOSTEP) and the Academy of Finland (project 272157, Center of Excellence ReSoLVE). The authors would like to thank the Editor and anonymous reviewers for their insightful comments and suggestions that have contributed to improve this paper.

\section{References}

Al Anid, H., Lewis, B., Bennett, L., Takada, M., Duldig, M., 2014. Aircrew radiation dose estimates during recent solar particle events and the effect of particle anisotropy. Radiation Protection Dosimetry 158 (3), 355-367.

Baker, D., 1998. What is space weather? Advances in Space Research 22 (1), 7-16.

Beck, P., Bartlett, D., Lindborg, L., McAulay, I., Schnuer, K., Schraube, H., 
Spurny, F., 2006. Aircraft crew radiation workplaces: Comparison of measured and calculated ambient dose equivalent rate data using the eurados in-flight radiation data base. Radiation Protection Dosimetry 118 (2), 182189.

Belov, A., Eroshenko, E., Mavromichalaki, H., Plainaki, C., Yanke, V., 2005. Solar cosmic rays during the extremely high ground level enhancement on 23 February 1956. Annales Geophysicae 23 (6), 2281-2291.

Bieber, J., Clem, J., Evenson, P., Pyle, R., Siz, A., Ruffolo, D., 2013. Giant ground level enhancement of relativistic solar protons on 2005 January 20. i. spaceship earth observations. Astrophysical Journal 771 (2), 92.

Bieber, J., Evenson, P., 1991. Determination of energy spectra for the large solar particle events of 1989. In: Proc. of 22th ICRC Dublin, Ireland, 11-23 August, 1991. Vol. 3. pp. 129-132.

Bombardieri, D., Duldig, M., Humble, J., Michael, K., 2008. An improved model for relativistic solar proton acceleration applied to the 2005 January 20 and earlier events. Astrophysical Journal 682 (2), 1315-1327.

Bombardieri, D., Duldig, M., Michael, K., Humble, J., 2006. Relativistic proton production during the 2000 July 14 solar event: The case for multiple source mechanisms. Astrophysical Journal 644 (1), 565-574.

Bombardieri, D., Michael, K., Duldig, M., Humble, J., 2007. Relativistic proton production during the 2001 April 15 solar event. Astrophysical Journal 665 (1 Part 1), 813-823.

Bottollier-Depois, J., Beck, P., Bennett, B., Bennett, L., Bütikofer, R., Clairand, I., Desorgher, L., Dyer, C., Felsberger, E., Flückiger, E., Hands, A., Kindl, P., Latocha, M., Lewis, B., Leuthold, G., Maczka, T., Mares, V., McCall, M., O’Brien, K., Rollet, S., Rühm, W., Wissmann, F., 2009. Comparison of codes assessing galactic cosmic radiation exposure of aircraft crew. Radiation Protection Dosimetry 136 (4), 317-323.

Burger, R., Potgieter, M., Heber, B., 2000. Rigidity dependence of cosmic ray proton latitudinal gradients measured by the Ulysses spacecraft: Implication for the diffusion tensor. Journal of Geophysical Research 105, 27447-27445.

Bütikofer, R., Flückiger, E., 2013. Differences in published characteristics of GLE 60 and their consequences on computed radiation dose rates along selected flight paths. Journal of Physics: Conference Series 409 (1), 012166.

Bütikofer, R., Flückiger, E., 2015. What are the causes for the spread of GLE parameters deduced from NM data? Journal of Physics: Conference Series $632(1)$.

Bütikofer, R., Flückiger, E., Desorgher, L., Moser, M., Pirard, B., 2009. The solar cosmic ray ground-level enhancements on 20 January 2005 and 13 December 2006. Advances in Space Research 43 (4), 499-503.

Copeland, K., 2017. Cari-7a: Development and validation. Radiation Protection Dosimetry 175 (4), 419-431.

Cramp, J., Duldig, M., Flückiger, E., Humble, J., Shea, M., Smart, D., 1997a. The October 22, 1989, solar cosmic enhancement: ray an analysis the anisotropy spectral characteristics. Journal of Geophysical Research 
102 (A11), 24 237-24 248.

Cramp, J., Duldig, M., Humble, J., 1993. The GLE of 29 September 1989. In: Proc. of 23th ICRC 19-30 July 1993, Calgary, Canada. Vol. 3. pp. 47-50.

Cramp, J., Duldig, M., Humble, J., 1997b. The effect of a distorted interplanetary magnetic field configuration on the December 7-8, 1982, ground level enhancement. Journal of Geophysical Research A: Space Physics 102 (A3), 4919-4925.

Cramp, J., Humble, J., Duldig, M., 1995. The cosmic ray ground-level enhancement of 24 October 1989. In: Proceedings Astronomical Society of Australia. Vol. 11. pp. 28-32.

Debrunner, H., Flückiger, E., Lockwood, J., McGuire, R., 1984. Comparison of the solar cosmic ray events on may 7, 1978, and November 22, 1977. Journal of Geophysical Research 89 (A2), 769-774.

Debrunner, H., Lockwood, J. A., 1980. The spatial anisotropy, rigidity spectrum, and propagation characteristics of the relativistic solar particles during the event on may 7, 1978. Journal of Geophysical Research: Space Physics 85 (A12), 6853-6860.

Deeley, K., Duldig, M., Humble, J., 2002. Re-analysis of the cosmic ray ground level enhancement of 4 May 1960. Advances in Space Research 30 (4), 1049 1052.

Desai, M., Giacalone, J., 2016. Large gradual solar energetic particle events. Living Reviews in Solar Physics 13 (1), 3.

EURATOM, 1996. Council directive 96/29/EURATOM of 13 May 1996 laying down basic safety standards for protection of the health of workers and the general public against the dangers arising from ionising radiation. Official Journal of the European Communities 39 (L159).

EURATOM, 2013. Council directive 2013/59/EURATOM of 5 December 2013 laying down basic safety standards for protection against the dangers arising from exposure to ionising radiation, and repealing Directives 89/618/Euratom, 90/641/Euratom, 96/29/Euratom, 97/43/Euratom and 2003/122/Euratom (L13).

Ferrari, A., Pelliccioni, M., Rancati, T., 2001. Calculation of the radiation environment caused by galactic cosmic rays for determining air crew exposure. Radiation Protection Dosimetry 93 (2), 101-114.

Gaisser, T. K., Stanev, T., 2010. Cosmic rays. in: Nakamura K. N. et al., Review of Particle Physics. Journal of Physics G 37, 269-275.

Getley, I., Duldig, M., Smart, D., Shea, M., 2005a. The applicability of model based aircraft radiation dose estimates. Advances in Space Research 36 (9), $1638-1644$.

Getley, I. L., 2004. Observation of solar particle event on board a commercial flight from los angeles to new york on 29 october 2003. Space Weather 2 (5), S05002.

Getley, I. L., Duldig, M. L., Smart, D. F., Shea, M. A., 2005b. Radiation dose along north American transcontinental flight paths during quiescent and disturbed geomagnetic conditions. Space Weather 3 (1), S01004. 
Gleeson, L., Axford, W., 1968. Solar modulation of galactic cosmic rays. Astrophysical Journal 154, 1011-1026.

Gopalswamy, N., Xie, H., Yashiro, S., Akiyama, S., Mäkelä, P., Usoskin, I., 2012. Properties of ground level enhancement events and the associated solar eruptions during solar cycle 23. Space Science Reviews 171 (1-4), 23-60.

Hands, A., Lei, F., Ryden, K., Dyer, C., Underwood, C., Mertens, C., 2017. New data and modelling for single event effects in the stratospheric radiation environment. IEEE Transactions on Nuclear Science 64 (1), 587-595.

Humble, J., Duldig, M., Smart, D., Shea, M., 1991. Detection of $0.515 \mathrm{GeV}$ solar protons on 29 September 1989 at Australian stations. Geophysical Research Letters 18 (4), 737-740.

ICRP, 1991. ICRP publication 60: 1990 recommendations of the international commission on radiological protection. Annals of the ICRP 21 (1-3).

ICRP, 2007. ICRP Publication 103: The 2007 Recommendations of the International Commission on Radiological Protection. Annals of the ICRP $37(2-4)$.

Kataoka, R., Sato, T., Kubo, Y., Shiota, D., Kuwabara, T., Yashiro, S., Yasuda, H., 2014. Radiation dose forecast of wasavies during ground-level enhancement. Space Weather 12 (6), 380-386.

Klein, K.-L., Dalla, S., 2017. Acceleration and propagation of solar energetic particles. Space Science Reviews 212 (3-4), 1107-1136.

Kocharov, L., Pohjolainen, S., Mishev, A., Reiner, M., Lee, J., Laitinen, T., Didkovsky, L., Pizzo, V., Kim, R., Klassen, A., Karlicky, M., Cho, K.-S., Gary, D., Usoskin, I., Valtonen, E., Vainio, R., 2017. Investigating the origins of two extreme solar particle events: Proton source profile and associated electromagnetic emissions. Astrophysical Journal 839 (2), 79.

Kravtsova, M. V., Sdobnov, V. E., 2016. Ground level enhancement of cosmic rays on November 6, 1997: Spectra and anisotropy. JETP Letters 103 (1), 8-14.

Latocha, M., Beck, P., Rollet, S., 2009. Avidos-a software package for European accredited aviation dosimetry. Radiation Protection Dosimetry 136 (4), 286-290.

Lilensten, L., Bornarel, J., 2009. Space Weather, Environment and Societies. Springer, Dordrecht.

Lockwood, J., Debrunner, H., Flükiger, E., Grädel, H., 1990. Proton energy spectra at the sun in the solar cosmic-ray events on 1978 May 7 and 1984 February 16. Astrophysical Journal 355 (1), 287-294.

Lovell, J., Duldig, M., Humble, J., Shea, M., Smart, D., Flckiger, E., 2002. The cosmic ray ground level enhancement of 6 November 1997. Advances in Space Research 30 (4), 1045-1048.

Lovell, J. L., Duldig, M. L., Humble, J. E., 1998. An extended analysis of the September 1989 cosmic ray ground level enhancement. Journal of Geophysical Research: Space Physics 103 (A10), 23733-23742.

Matthiä, D., Heber, B., Reitz, G., Meier, M., Sihver, L., Berger, T., Herbst, K., 2009a. Temporal and spatial evolution of the solar energetic particle 
event on 20 January 2005 and resulting radiation doses in aviation. Journal of Geophysical Research: Space Physics 114 (8).

Matthiä, D., Heber, B., Reitz, G., Sihver, L., Berger, T., Meier, M., 2009b. The ground level event 70 on December 13th, 2006 and related effective doses at aviation altitudes. Radiation Protection Dosimetry 136 (4), 304-310.

Matthiä, D., Sihver, L., Meier, M., 2008. Monte-Carlo calculations of particle fluences and neutron effective dose rates in the atmosphere. Radiation Protection Dosimetry 131 (2), 222-228.

Menzel, H. The international commission on radiation units and measurements. Journal of the ICRU, 10(2), 1-35, 2010.

Mertens, C., Meier, M., Brown, S., Norman, R., Xu, X., 2013. Nairas aircraft radiation model development, dose climatology, and initial validation. Space Weather 11 (10), 603-635.

Miroshnichenko, L., Klein, K.-L., Trottet, G., Lantos, P., Vashenyuk, E., Balabin, Y., Gvozdevsky, B., 2005. Relativistic nucleon and electron production in the 2003 October 28 solar event. Journal of Geophysical Research: Space Physics 110 (A9), A09S08.

Mishev, A., Adibpour, F., Usoskin, I., Felsberger, E., 2014a. Computation of dose rate at flight altitudes during ground level enhancements no. 69, 70 and 71. Advances in Space Research 55 (1), 354-362.

Mishev, A., Kocharov, L., Usoskin, I., 2014b. Analysis of the ground level enhancement on 17 May 2012 using data from the global neutron monitor network. Journal of Geophysical Research 119, 670-679.

Mishev, A., Usoskin, I., 2015. Numerical model for computation of effective and ambient dose equivalent at flight altitudes: Application for dose assessment during GLEs. Journal of Space Weather and Space Climate 5 (3), A10.

Mishev, A., Usoskin, I., 2016. Analysis of the ground level enhancements on 14 July 2000 and on 13 December 2006 using neutron monitor data. Solar Physics 291 (4), 1225-1239.

Mishev, A., Velinov, P., 2011. Normalized ionization yield function for various nuclei obtained with full Monte Carlo simulations. Advances of Space Research 48 (1), 19-24.

Moraal, H., McCracken, K., 2012. The time structure of ground level enhancements in solar cycle 23. Space Science Reviews 171 (1-4), 85-95.

O'Brien, K., 1970. Calculated cosmic ray ionization in the lower atmosphere. Journal of Geophysical Research 75 (22), 4357-4359.

Petoussi-Henss, N., Bolch, W., Eckerman, K., Endo, A., Hertel, N., Hunt, J., Pelliccioni, M., Schlattl, H., Zankl, M., 2010. Conversion coefficients for radiological protection quantities for external radiation exposures. Annals of the ICRP 40 (2-5), 1-257.

Plainaki, C., Mavromichalaki, H., Laurenza, M., Gerontidou, M., Kanellakopoulos, A., Storini, M., 2014. The ground-level enhancement of 2012 May 17: Derivation of solar proton event properties through the application of the NMBANGLE PPOLA model. Astrophysical Journal 785 (2), 160. 
Ploc, O., Ambrozova, I., Kubancak, J., Kovar, I., Dachev, T., 2013. Publicly available database of measurements with the silicon spectrometer liulin onboard aircraft. Radiation Measurements 58, 107-112.

Reames, D., 2013. The two sources of solar energetic particles. Space Science Reviews 175 (1-4), 53-92.

Sato, T., Yasuda, H., Niita, K., Endo, A., Sihver, L., 2008. Development of parma: Phits-based analytical radiation model in the atmosphere. Radiation Research 170, 244-259.

Shea, M., Smart, D., 1982. Possible evidence for a rigidity-dependent release of relativistic protons from the solar corona. Space Science Reviews 32, $251-271$.

Shea, M., Smart, D., 1990. A summary of major solar proton events. Solar Physics 127, 297-320.

Shea, M., Smart, D., 2000. Cosmic ray implications for human health. Space Science Reviews 93 (1-2), 187-205.

Smart, D., Shea, M., 1994. The relativistic solar proton groundlevel enhancements associated with the solar neutron events of 11 June, 15 June 1991. AIP Conference Proceedings 294, 222-229.

Smart, D., Shea, M., Gentile, L., 1993. The relativistic solar proton event of 15 june 1991. In: Proc. of 23th ICRC 19-30 July 1993, Calgary, Canada. Vol. 3., pp. 59-62.

Spurny, F., Dachev, T., 2001. Measurements in an aircraft during an intense solar flare, ground level event 60, on april 15, 2001. Radiation Protection Dosimetry 95 (3), 273-275.

Spurny, F., I.Votockova, Bottollier-Depois, J., 1996. Geographical influence on the radiation exposure of an aircrew on board a subsonic aircraft. Radioprotection 31 (2), 275-280.

Spurny, F., T.Dachev, Kudela, K., 2002. Increase of onboard aircraft exposure level during a solar flare. Nuclear Energy Safety 10 (48), 396-400.

Stoker, P., 1995. Relativistic solar proton events. Space Science Reviews 73 (34), 327-385.

Usoskin, I., Bazilevskaya, G., Kovaltsov, G., 2011. Solar modulation parameter for cosmic rays since 1936 reconstructed from ground-based neutron monitors and ionization chambers. Journal of Geophysical Research 116, A02104.

Usoskin, I., Ibragimov, A., Shea, M., Smart, D., 2015. Database of ground level enhancements (GLE) of high energy solar proton events. Proceedings of Science, Proc. of 34th ICRC Hague, Netherlands, 30 July - 6 August 2015 30-July-2015, 054.

Usoskin I.G., Alanko-Huotari K., Kovaltsov G.A., Mursula K., 2005. Heliospheric modulation of cosmic rays: Monthly reconstruction for 1951-2004. Journal of Geophysical Research 110, A12108.

Usoskin, I., Kovaltsov, G., 2006. Cosmic ray induced ionization in the atmosphere: Full modeling and practical applications. Journal of Geophysical Research 111 (D21206). 
Vainio, R., Desorgher, L., Heynderickx, D., Storini, M., Flückiger, E., Horne, R., Kovaltsov, G., Kudela, K., Laurenza, M., McKenna-Lawlor, S., Rothkaehl, H., Usoskin, I., 2009. Dynamics of the earth's particle radiation environment. Space Science Reviews 147 (3-4), 187-231.

Vashenyuk, E., Balabin, Y., Gvozdevskii, B., Karpov, S., 2006a. Relativistic solar protons in the event of January 20, 2005: Model studies. Geomagnetism and Aeronomy 46 (4), 424-429.

Vashenyuk, E., Balabin, Y., Gvozdevsky, B., 2011. Features of relativistic solar proton spectra derived from ground level enhancement events (GLE) modeling. Astrophysics and Space Sciences Transactions 7 (4), 459-463.

Vashenyuk, E., Balabin, Y., Miroshnichenko, L., 2008. Relativistic solar protons in the ground level event of 23 February 1956: New study. Advances in Space Research 41 (6), 926-935.

Vashenyuk, E., Balabin, Y., Perez-Peraza, J., Gallegos-Cruz, A., Miroshnichenko, L., 2006b. Some features of the sources of relativistic particles at the sun in the solar cycles 21-23. Advances Space Research 38 (3), 411417.

Xapsos, M., Stauffer, C., Jordan, T., Barth, J., Mewaldt, R., 2007. Model for cumulative solar heavy ion energy and linear energy transfer spectra. IEEE Transactions on Nuclear Science 54 (6), 1985-1989. 
Table 1

List of GLE events used for the upgrade of the GLE database gle.oulu.fi with the corresponding date and bibliography.

\begin{tabular}{|c|c|c|}
\hline GLE & Date & Bibliography \\
\hline 5 & 23.021956 & (Belov et al., 2005; Vashenyuk et al., 2008, 2011) \\
\hline 8 & 04.051960 & (Deeley et al., 2002; Vashenyuk et al., 2011) \\
\hline 10 & 12.111960 & (Vashenyuk et al., 2011) \\
\hline 11 & 15.111960 & (Vashenyuk et al., 2011) \\
\hline 13 & 18.071961 & (Vashenyuk et al., 2011) \\
\hline 16 & 28.011967 & (Vashenyuk et al., 2011) \\
\hline 19 & 18.111968 & (Vashenyuk et al., 2011) \\
\hline 22 & 14.011971 & (Vashenyuk et al., 2011) \\
\hline 25 & 07.081972 & (Vashenyuk et al., 2011) \\
\hline 29 & 24.091977 & (Vashenyuk et al., 2011) \\
\hline 30 & 22.111977 & (Debrunner et al., 1984; Vashenyuk et al., 2011) \\
\hline 31 & 07.051978 & (Debrunner and Lockwood, 1980; Debrunner et al., 1984; Lockwood et al., 1990; Vashenyuk et al., 2006b, 2011) \\
\hline 32 & 23.091978 & (Vashenyuk et al., 2011) \\
\hline 38 & 08.121982 & (Cramp et al., 1997b; Vashenyuk et al., 2006b, 2011) \\
\hline 39 & 16.021984 & (Vashenyuk et al., 2006b, 2011) \\
\hline 41 & 16.081989 & (Vashenyuk et al., 2011) \\
\hline 42 & 29.091989 & (Humble et al., 1991; Cramp et al., 1993; Vashenyuk et al., 2006b, 2011) \\
\hline 43 & 19.101989 & (Bieber and Evenson, 1991; Vashenyuk et al., 2006b, 2011) \\
\hline 44 & 22.101989 & (Cramp et al., 1997a; Vashenyuk et al., 2006b, 2011) \\
\hline 45 & 24.101989 & (Cramp et al., 1995; Lovell et al., 1998; Vashenyuk et al., 2011) \\
\hline 47 & 21.051990 & (Vashenyuk et al., 2011) \\
\hline 48 & 24.051990 & (Vashenyuk et al., 2011) \\
\hline 51 & 11.061991 & (Smart and Shea, 1994; Vashenyuk et al., 2011) \\
\hline 52 & 15.061991 & (Smart et al., 1993; Vashenyuk et al., 2011) \\
\hline 55 & 06.111997 & (Lovell et al., 2002; Vashenyuk et al., 2011; Kravtsova and Sdobnov, 2016) \\
\hline 59 & 14.072000 & (Bombardieri et al., 2006; Vashenyuk et al., 2006b, 2011; Mishev and Usoskin, 2016) \\
\hline 60 & 15.042001 & (Bombardieri et al., 2007; Vashenyuk et al., 2006b, 2011) \\
\hline 61 & 18.042001 & (Vashenyuk et al., 2011) \\
\hline 65 & 28.102003 & (Miroshnichenko et al., 2005; Vashenyuk et al., 2006b, 2011) \\
\hline 67 & 02.112003 & (Vashenyuk et al., 2006b, 2011; Kocharov et al., 2017) \\
\hline 69 & 20.012005 & $\begin{array}{l}\text { (Vashenyuk et al., 2006b,a; Bombardieri et al., 2008; Bütikofer et al., 2009) } \\
\text { (Matthiä et al., 2009a; Vashenyuk et al., 2011; Bieber et al., 2013) }\end{array}$ \\
\hline 70 & 13.122006 & (Bütikofer et al., 2009; Matthiä et al., 2009b; Vashenyuk et al., 2011; Mishev and Usoskin, 2016),(nmdb.eu) \\
\hline 71 & 17.052012 & (Mishev et al., 2014b; Plainaki et al., 2014),(nmdb.eu) \\
\hline
\end{tabular}


Table 2

GLE events, peak effective dose rate and contribution of GCRs to the exposure with the corresponding modulation potential.

\begin{tabular}{|c|c|c|c|c|}
\hline GLE & Date & $\max . E\left[\mu \mathrm{Sv}^{\prime} \mathrm{h}^{-1}\right]$ & $E_{G C R}\left[\mu{\left.\mathrm{Sv} \cdot \mathrm{h}^{-1}\right]}^{-1}\right.$ & Modulation potential [MV] \\
\hline 5 & 23.021956 & 2977 & 6.9 & 551 \\
\hline 8 & 04.051960 & 57.3 & 5.0 & 1109 \\
\hline 10 & 12.111960 & 12.1 & 5.2 & 1024 \\
\hline 11 & 15.111960 & 140.5 & 5.2 & 1024 \\
\hline 13 & 18.071961 & 13.7 & 5.4 & 956 \\
\hline 16 & 28.011967 & 15.8 & 6.4 & 632 \\
\hline 19 & 18.111968 & 11.4 & 5.3 & 985 \\
\hline 22 & 14.011971 & 25.1 & 6.2 & 717 \\
\hline 25 & 07.081972 & 7.8 & 6.4 & 636 \\
\hline 29 & 24.091977 & 8.8 & 7.3 & 475 \\
\hline 30 & 22.111977 & 15.5 & 7.7 & 408 \\
\hline 31 & 07.051978 & 35.4 & 6.4 & 669 \\
\hline 32 & 23.091978 & 8.1 & 7.2 & 495 \\
\hline 38 & 08.121982 & 22.4 & 4.7 & 1256 \\
\hline 39 & 16.021984 & 13.5 & 6.1 & 736 \\
\hline 41 & 16.081989 & 10.8 & 5.0 & 1114 \\
\hline 42 & 29.091989 & 92.7 & 4.8 & 1195 \\
\hline 43 & 19.101989 & 41.9 & 4.5 & 1356 \\
\hline 44 & 22.101989 & 92.5 & 4.5 & 1356 \\
\hline 45 & 24.101989 & 61.0 & 4.5 & 1356 \\
\hline 47 & 21.051990 & 12.0 & 4.3 & 1452 \\
\hline 48 & 24.051990 & 17.0 & 4.3 & 1452 \\
\hline 51 & 11.061991 & 6.0 & 3.5 & 2016 \\
\hline 52 & 15.061991 & 11.2 & 3.5 & 2016 \\
\hline 55 & 06.111997 & 19.9 & 7.5 & 439 \\
\hline 59 & 14.072000 & 48.1 & 4.9 & 1167 \\
\hline 60 & 15.042001 & 51.3 & 5.3 & 995 \\
\hline 61 & 18.042001 & 9.0 & 5.3 & 995 \\
\hline 65 & 28.102003 & 12.4 & 5.4 & 963 \\
\hline 67 & 02.112003 & 15.6 & 4.6 & 1281 \\
\hline 69 & 20.012005 & 3592 & 5.9 & 788 \\
\hline 70 & 13.122006 & 78.2 & 7.4 & 467 \\
\hline 71 & 17.052012 & 32.9 & 7.2 & 494 \\
\hline
\end{tabular}


Figure captions:

Fig.1 Screen shot of the stored information about GLE 59 on 14 July 2000 in http://gle.oulu.fi/\#/dose, namely the computed radiation doses at the cruise flight altitude of $35 \mathrm{kft}(10668 \mathrm{~m})$ for several different sets of derived spectra and the used bibliography. The numbering of the sets corresponds to the used references given on the bottom.

Fig.2 Screen shot of the stored information about GLE 60 on 15 April 2001 in http://gle.oulu.fi/\#/dose, namely the computed radiation doses at the cruise flight altitude of $35 \mathrm{kft}(10668 \mathrm{~m})$ and the used bibliography (on the bottom).

Fig.3 Screen shot of the stored information about GLE 70 on 13 December 2006 in http://gle.oulu.fi/\#/dose, namely the computed radiation doses at the cruise flight altitude of $35 \mathrm{kft}(10668 \mathrm{~m})$ for several different sets of derived spectra and the used bibliography. The numbering of the sets corresponds to the used references given on the bottom.

Fig.4 SEP spectra used for computation of radiation doses at the cruise flight altitude of $35 \mathrm{kft}(10668 \mathrm{~m})$ during GLE 59 on 14 July 2000. Panel A corresponds to set 3 , while panel B corresponds to set 1 .

Fig.5 SEP spectra used for computation of radiation doses at the cruise flight altitude of $35 \mathrm{kft}$ (10668 m) during GLE 60 on 15 April 2001.

Fig.6 SEP spectra used for computation of radiation doses at the cruise flight altitude of $35 \mathrm{kft}(10668 \mathrm{~m})$ during GLE 70 on 13 December 2006. Panel A corresponds to set 4 , while panel B corresponds to set 3 .

Fig.7 Hardest SEP spectra used for computation of radiation doses at the cruise flight altitude of $35 \mathrm{kft}(10668 \mathrm{~m})$ during GLE 59 on 20 January 2005. The spectra correspond to set 2 in the database. 


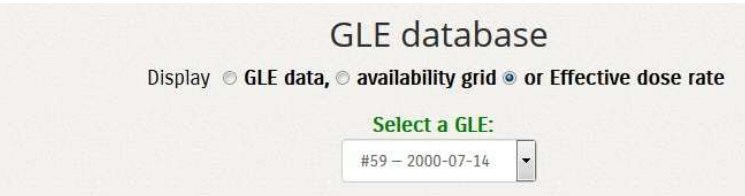

GLE: 59 at 2000-07-14

Modulation potential $1167 \mathrm{MV}$. Altitude $35 \mathrm{kft}$. GCR contribution $4.86 \mu \mathrm{Sv} \mathrm{h} \mathrm{h}^{-1}$.

\section{Effective dose rate, series \# 1}

\begin{tabular}{|ccc} 
Interval start [UTC] & Interval end [UTC] & Effective dose rate $\left[\boldsymbol{\mu S v} \mathbf{~ h}^{-1}\right.$ ] \\
\hline 2000-07-14 10:30:00 & N/A & 6.94 \\
\hline 2000-07-14 11:50:00 & N/A & 48.16 \\
\hline 2000-07-14 14:00:00 & N/A & 14.53 \\
\hline
\end{tabular}

Effective dose rate, series \# 2

\begin{tabular}{|ccc}
\hline Interval start [UTC] & Interval end [UTC] & Effective dose rate $\left[\boldsymbol{\mu S v} \mathbf{~}^{-1}\right]$ \\
\hline 2000-07-14 11:05:00 & N/A & 4.87 \\
\hline 2000-07-14 12:00:00 & N/A & 5.48 \\
\hline 2000-07-14 14:10:00 & N/A & 6.43 \\
\hline 2000-07-14 14:40:00 & N/A & 7.45 \\
\hline 2000-07-14 16:00:00 & N/A & 6.27 \\
\hline
\end{tabular}

Effective dose rate, series \# 3

\begin{tabular}{|ccc}
\hline Interval start [UTC] & Interval end [UTC] & Effective dose rate $\left[\boldsymbol{\mu S v} \mathbf{~ h}^{-1}\right.$ ] \\
\hline 2000-07-14 10:35:00 & $2000-07-14$ 10:40:00 & 18.51 \\
\hline 2000-07-14 10:45:00 & $2000-07-14$ 10:50:00 & 22.68 \\
\hline 2000-07-14 11:55:00 & $2000-07-14$ 12:00:00 & 14.92 \\
\hline 2000-07-14 15:55:00 & $2000-07-14$ 16:00:00 & 9.47 \\
\hline
\end{tabular}

\section{References}

[1] RELATIVISTIC PROTON PRODUCTION DURING THE 2000 JULY 14 SOLAR EVENT: THE CASE FOR MULTIPLE SOURCE MECHANISMS (Bombardieri)

[2] Relativistic Solar proton Dynamics in Large GLE of 23rd Solar Cycle (Vashenyuk)

[3] Analysis of the Ground-Level Enhancements on 14 July 2000 and 13 December 2006 Using Neutron Monitor Data (Mishev)

Fig. 1. Screen shot of the stored information about GLE 59 on 14 July 2000 in http://gle.oulu.fi/\#/dose, namely the computed radiation doses at the cruise flight altitude of $35 \mathrm{kft}(10668 \mathrm{~m})$ for several different sets of derived spectra and the used bibliography. The numbering of the sets corresponds to the used references given on the bottom. 


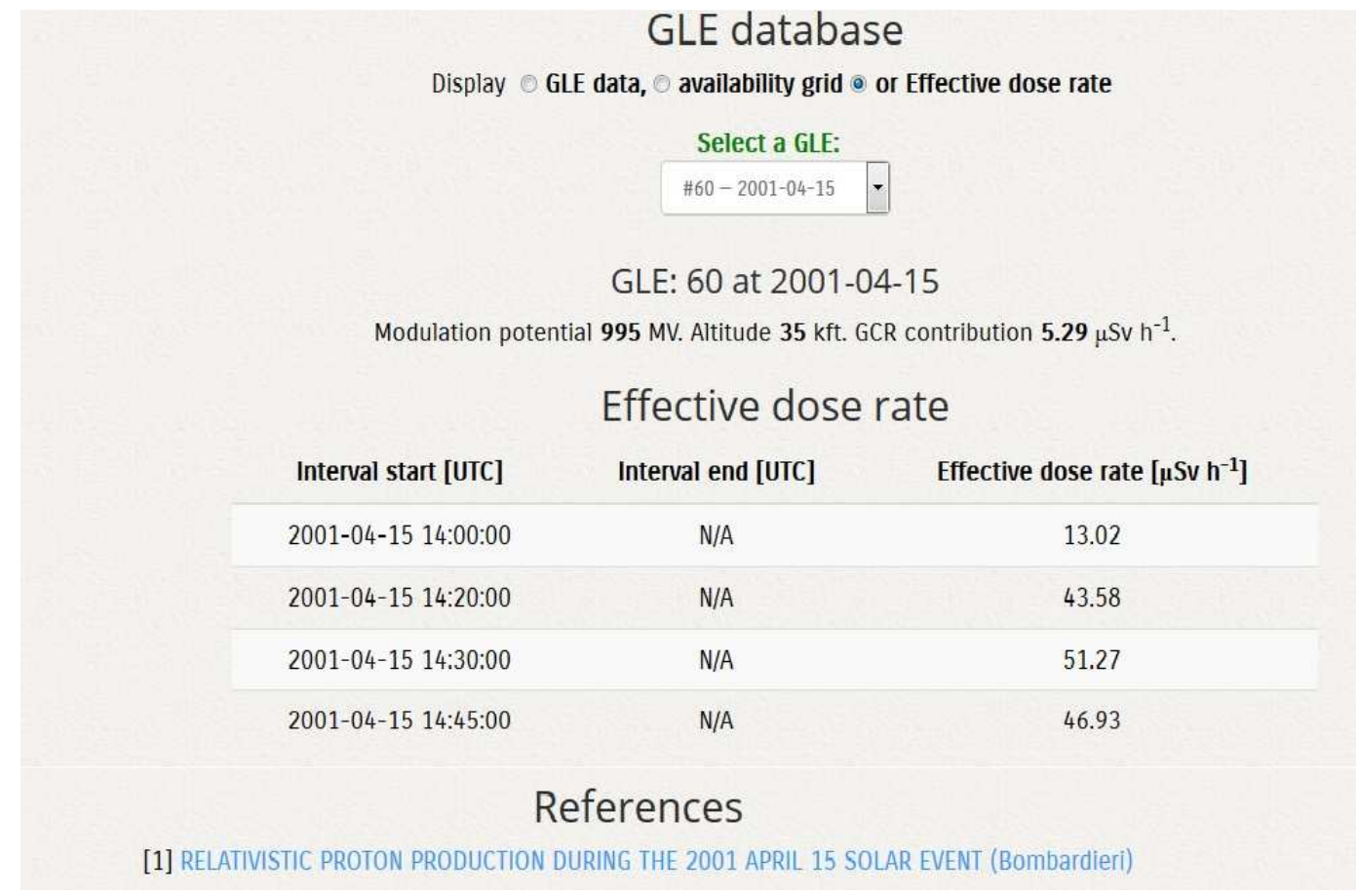

Fig. 2. Screen shot of the stored information about GLE 60 on 15 April 2001 in http://gle.oulu.fi/\#/dose, namely the computed radiation doses at the cruise flight altitude of $35 \mathrm{kft}$ (10668 m) and the used bibliography (on the bottom). 


\section{GLE database}

Display $\odot$ GLE data, $\odot$ availability grid $\odot$ or Effective dose rate

Select a GLE:

$\# 70-2006-12-13$

GLE: 70 at $2006-12-13$

Modulation potential $467 \mathrm{MV}$. Altitude $35 \mathrm{kft}$. GCR contribution $7.36 \mu \mathrm{Sv} \mathrm{h}^{-1}$.

\section{Effective dose rate, series \# 1}

\begin{tabular}{|c|c|c|}
\hline Interval start [UTC] & Interval end [UTC] & Effective dose rate $\left[\mu \mathrm{Sv} \mathrm{h}^{-1}\right]$ \\
\hline 2006-12-13 02:58:00 & N/A & 40.34 \\
\hline 2006-12-13 03:38:00 & $N / A$ & 19.58 \\
\hline 2006-12-13 04:38:00 & $N / A$ & 13.65 \\
\hline 2006-12-13 06:38:00 & $N / A$ & 9.41 \\
\hline
\end{tabular}

Effective dose rate, series \# 2

\begin{tabular}{ccc} 
Interval start [UTC] & Interval end [UTC] & Effective dose rate $\left[\boldsymbol{\mu S v ~} \mathbf{~ h}^{-1}\right.$ ] \\
\hline 2006-12-13 02:57:00 & N/A & 21.84 \\
\hline 2006-12-13 03:20:00 & N/A & 67.3 \\
2006-12-13 04:00:00 & N/A & 78.16
\end{tabular}

Effective dose rate, series \# 3

\begin{tabular}{ccc} 
Interval start [UTC] & Interval end [UTC] & Effective dose rate $\left[\boldsymbol{\mu S v} \mathbf{~ h}^{-\mathbf{1}}\right.$ ] \\
\hline 2006-12-13 03:00:00 & N/A & 17.55 \\
\hline 2006-12-13 04:30:00 & N/A & 21.98 \\
\hline 2006-12-13 05:30:00 & N/A & 4.43 \\
\hline
\end{tabular}

\section{Effective dose rate, series \# 4}

\begin{tabular}{|ccc|}
\hline Interval start [UTC] & Interval end [UTC] & Effective dose rate $\left[\boldsymbol{\mu S v} \mathbf{~ h}^{-\mathbf{1}}\right.$ ] \\
\hline 2006-12-13 03:00:00 & $2006-12-1303: 05: 00$ & 42.14 \\
\hline 2006-12-13 03:05:00 & $2006-12-1303: 10: 00$ & 43.54 \\
\hline 2006-12-13 03:35:00 & $2006-12-1303: 40: 00$ & 14.37 \\
\hline 2006-12-13 05:55:00 & $2006-12-1306: 00: 00$ & 9.4 \\
\hline
\end{tabular}

\section{Effective dose rate, series \# 5}

$\begin{array}{ccc}\text { Interval start [UTC] } & \text { Interval end [UTC] } & \text { Effective dose rate }\left[\mu \mathrm{Sv} \mathrm{h}^{-1} \text { ] }\right. \\ 2006-12-1300: 00: 00 & \mathrm{~N} / \mathrm{A} & 52.02\end{array}$

\section{References}

[1] THE GROUND LEVEL EVENT 70 ON DECEMBER 13TH, 2006 AND RELATED EFFECTIVE DOSES ATAVIATION ALTITUDES (Matthia) [2] The GLE of December 13, 2006 according to the ground level and balloon observations (Vashenyuk) [3] pgia.ru

[4] Analysis of the Ground-Level Enhancements on 14 July 2000 and 13 December 2006 Using Neutron Monitor Data (Mishev) [5] The solar cosmic ray ground-level enhancements on 20 January 2005 and 13 December 2006 (Butikofer)

Fig. 3. Screen shot of the stored information about GLE 70 on 13 December 2006 in http://gle.oulu.fi/\#/dose, namely the computed radiation doses at the cruise flight altitude of $35 \mathrm{kft}(10668 \mathrm{~m})$ for several different sets of derived spectra and the used bibliography. The numbering of the sets corresponds to the used references given on the bottom. 

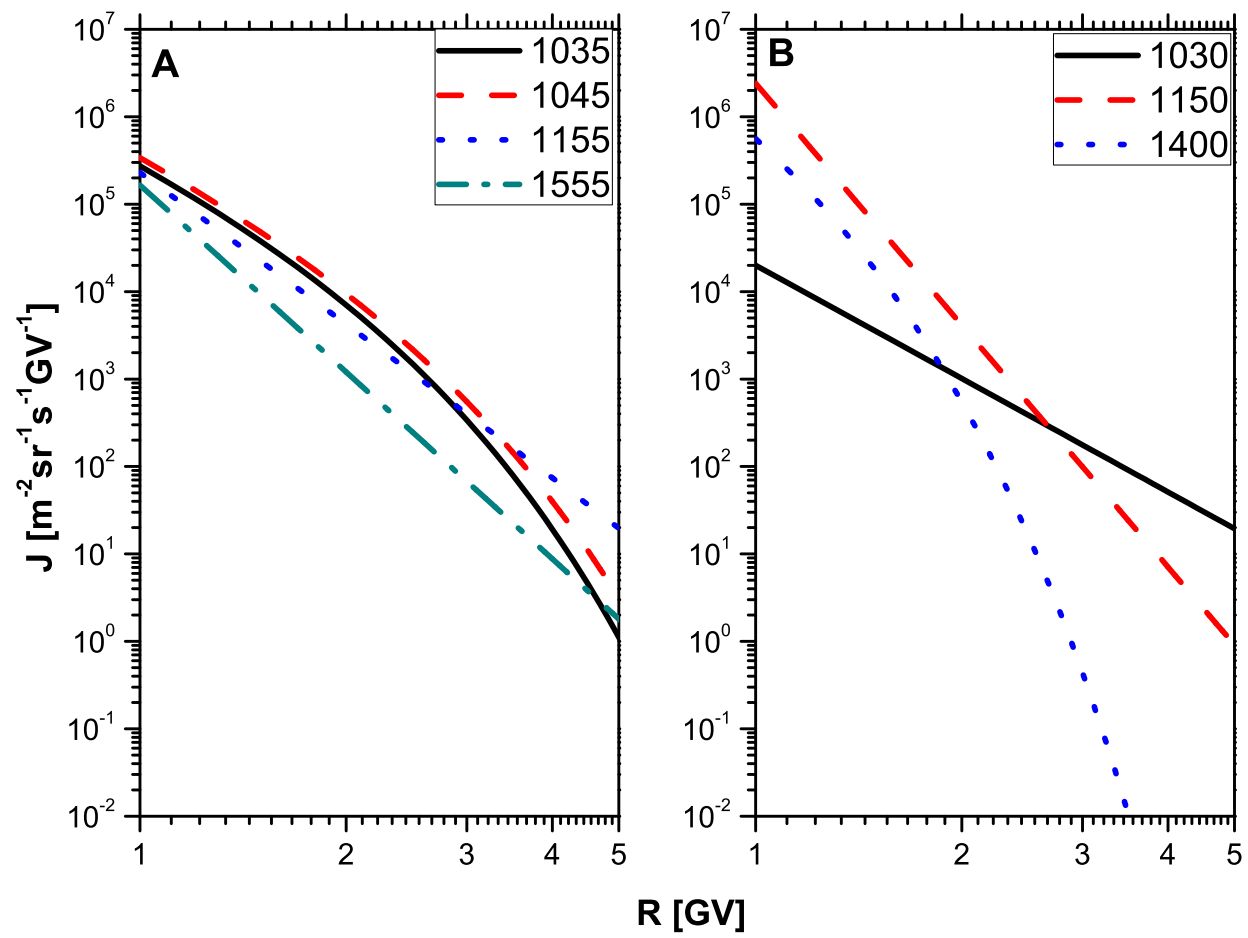

Fig. 4. SEP spectra used for computation of radiation doses at the cruise flight altitude of $35 \mathrm{kft}$ (10668 m) during GLE 59 on 14 July 2000. Panel A corresponds to set 3 , while panel B corresponds to set 1 . 


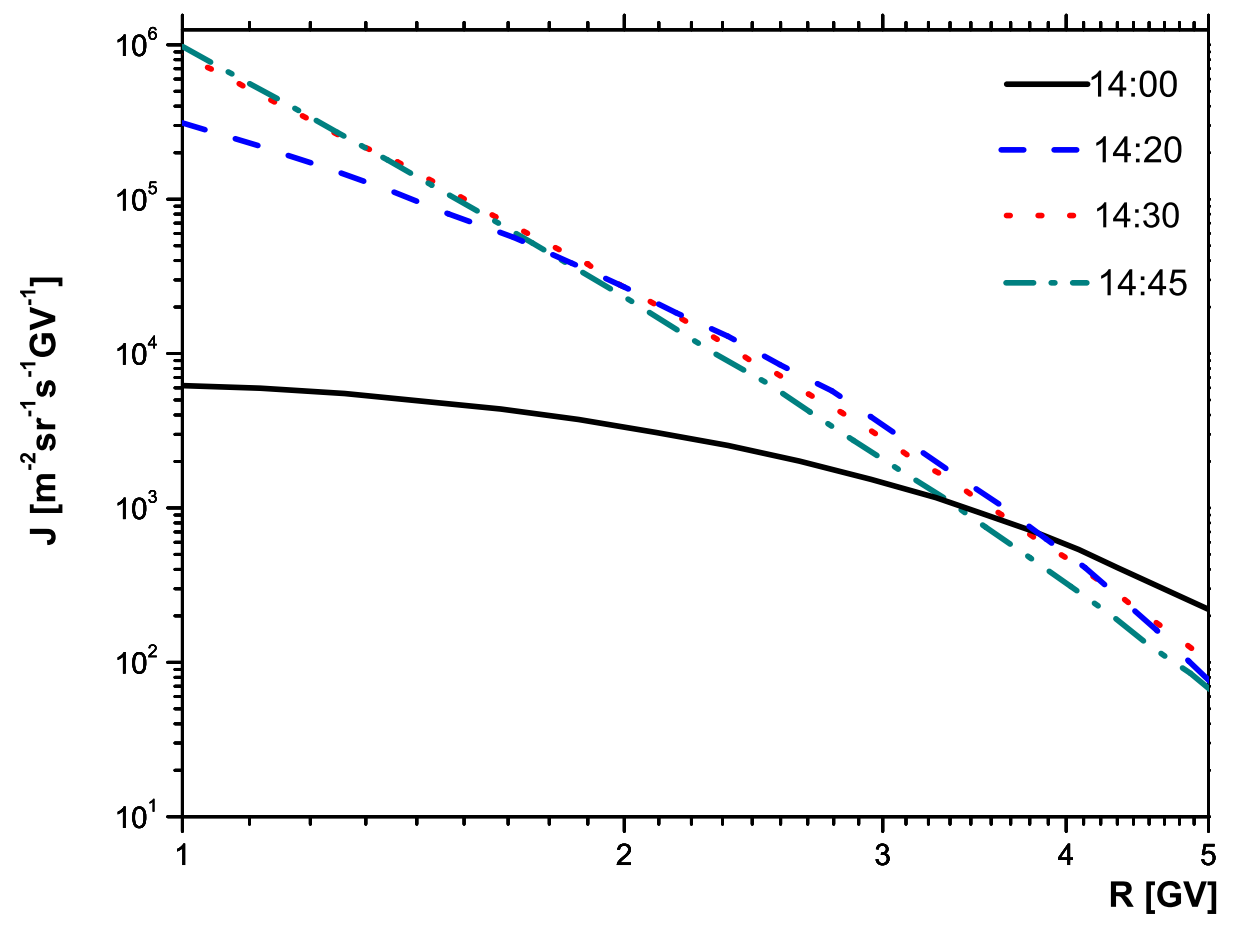

Fig. 5. SEP spectra used for computation of radiation doses at the cruise flight altitude of $35 \mathrm{kft}$ (10668 m) during GLE 60 on 15 April 2001. 

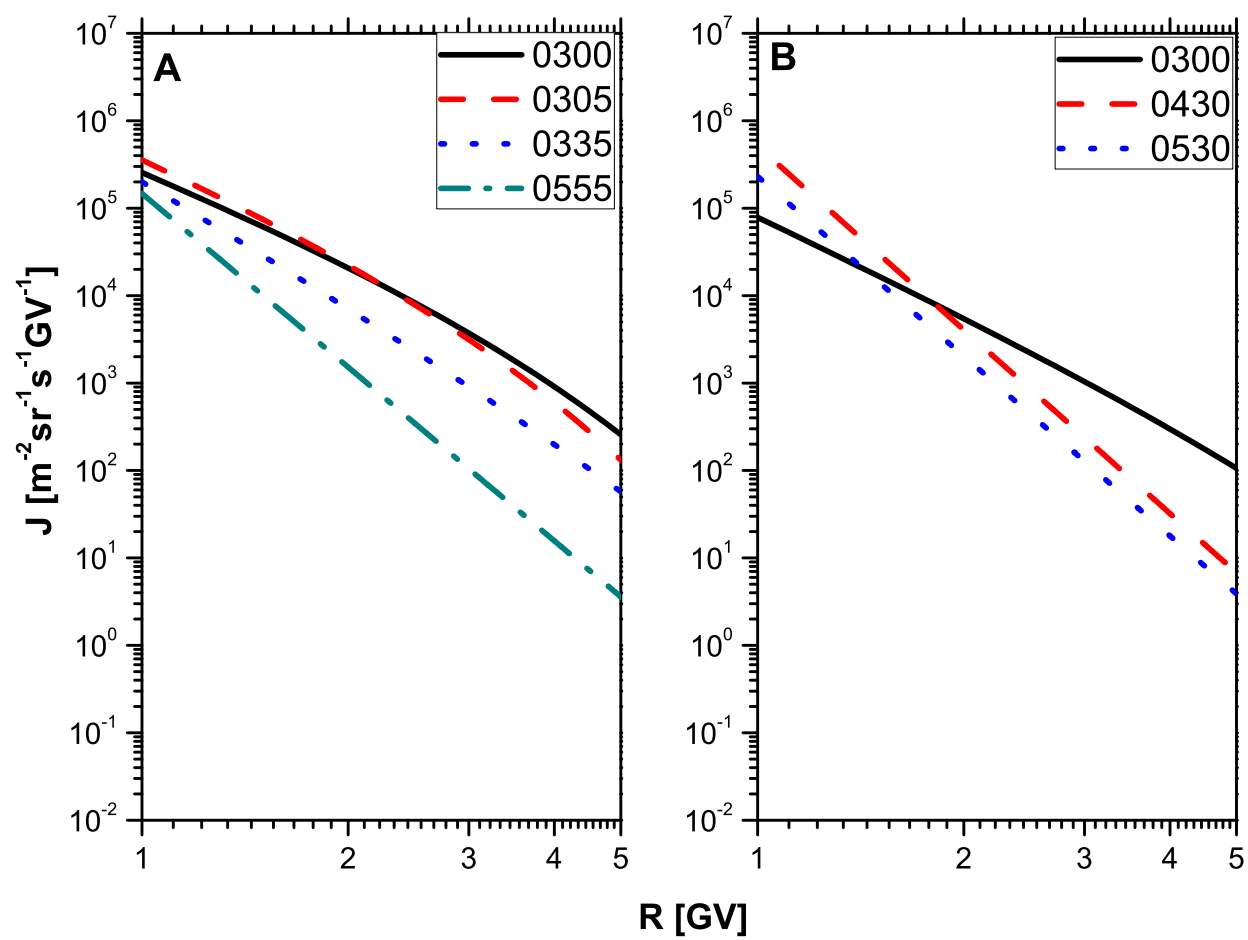

Fig. 6. SEP spectra used for computation of radiation doses at the cruise flight altitude of $35 \mathrm{kft}$ (10668 m) during GLE 70 on 13 December 2006. Panel A corresponds to set 4 , while panel B corresponds to set 3 . 


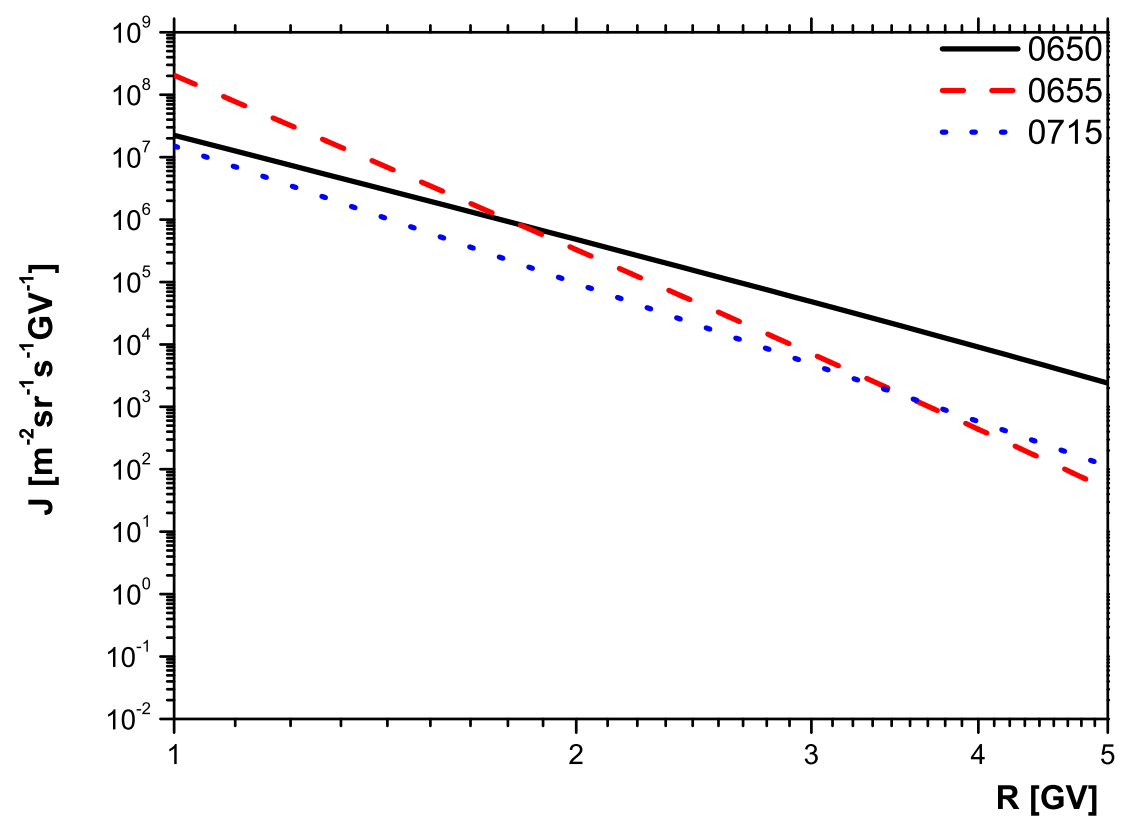

Fig. 7. Hardest SEP spectra used for computation of radiation doses at the cruise flight altitude of $35 \mathrm{kft}(10668 \mathrm{~m})$ during GLE 59 on 20 January 2005. The spectra correspond to set 2 in the database. 